SEMICONDUCTOR TECHNOLOGY FOR THE NON-TECHNOLOGIST, SECOND EDITION

\section{Robert I. Scace}

U.S. DEPARTMENT OF COMMERCE National Institute of Standards and Technology Center for Electronics and Electricai Engineering

Gaithersburg, MD 20899

September 1990

U.S. DEPARTMENT OF COMMERCE Robert A. Mosbacher, Secretary NATIONAL INSTITUTE OF STANDARDS AND TECHNOLOGY

John W. Lyons, Director

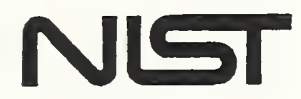





\section{SEMICONDUCTOR}

TECHNOLOGY FOR THE

NON-TECHNOLOGIST,

SECOND EDITION

\section{Robert I. Scace}

\author{
U.S. DEPARTMENT OF COMMERCE \\ National Institute of Standards \\ and Technology \\ Center for Electronics and Electrical \\ Englneering \\ Galthersburg, MD 20899
}

September 1990

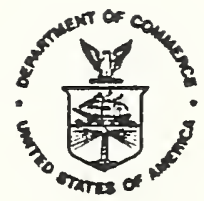

U.S. DEPARTMENT OF COMMERCE Robert A. Mosbacher, Secretary NATIONAL INSTTUTE OF STANDARDS AND TECHNOLOQY

John W. Lyons, Director 



\section{TABLE OF CONTENTS}

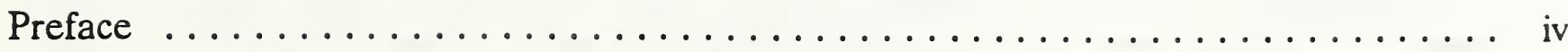

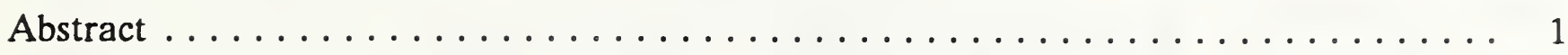

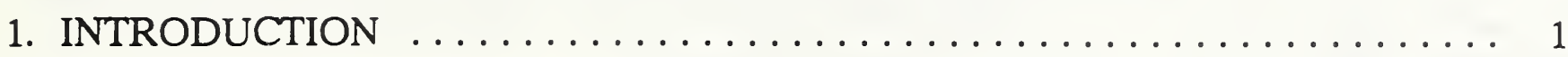

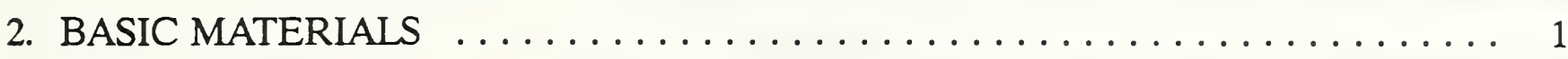

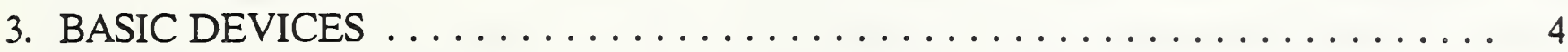

4. SEMICONDUCTOR MANUFACTURING EQUIPMENT $\ldots \ldots \ldots \ldots \ldots \ldots$

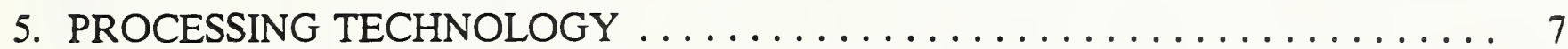

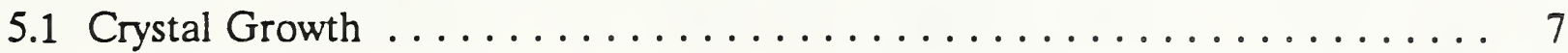

5.2 Mechanical Processes . . . . . . . . . . . . . . . . 9

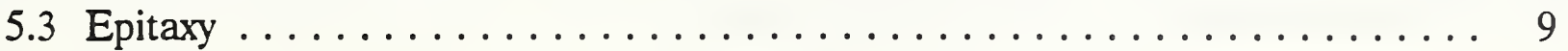

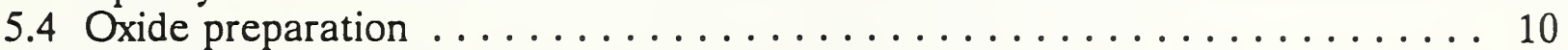

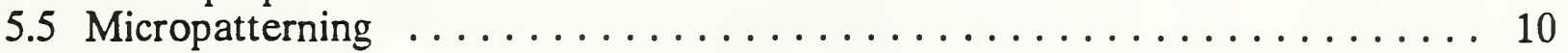

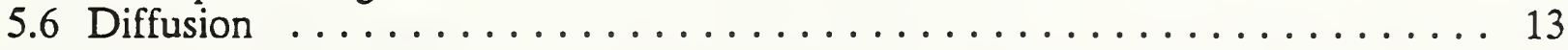

5.7 Ion Implantation $\ldots \ldots \ldots \ldots \ldots \ldots \ldots \ldots \ldots \ldots \ldots \ldots \ldots \ldots \ldots \ldots$

5.8 Passivation . . . . . . . . . . . . . . . . . 14

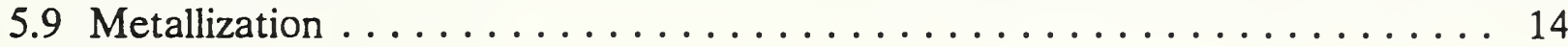

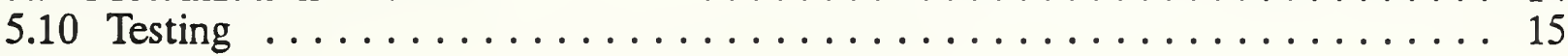

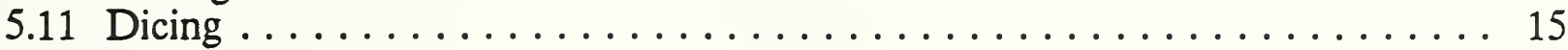

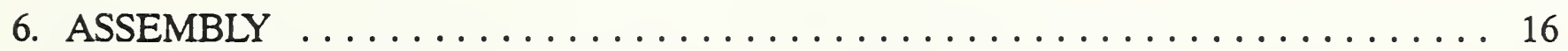

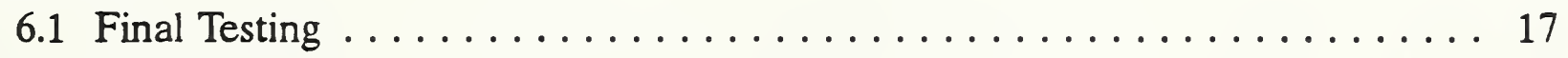

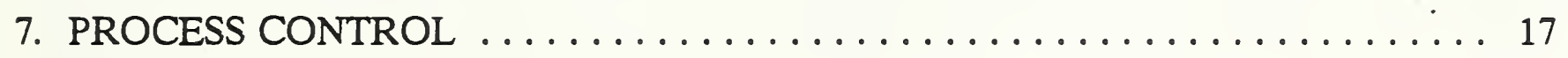

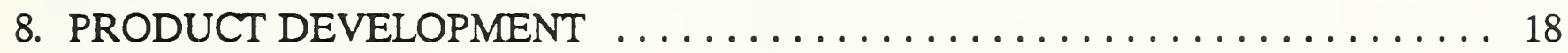

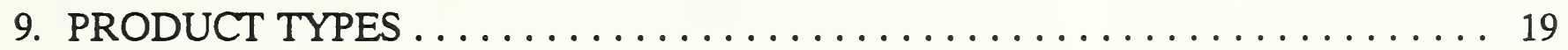

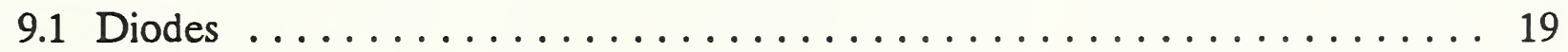

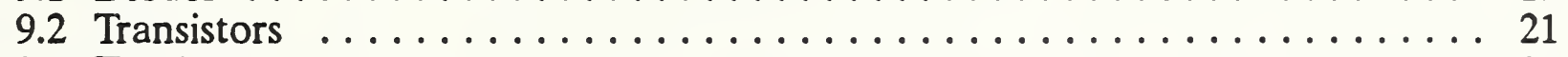

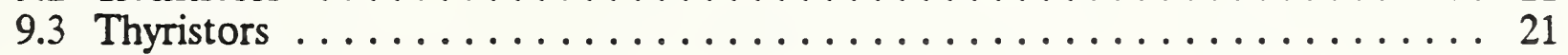

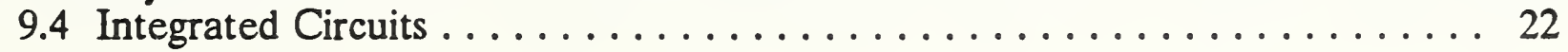

10. APPLICATION TRENDS AND THEIR IMPACTS .............. 24

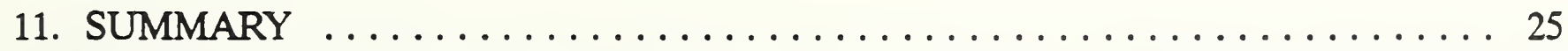

APPENDIX A. Units of Measurement ................... 26

APPENDIX B. How silicon is obtained $\ldots \ldots \ldots \ldots \ldots \ldots \ldots \ldots \ldots \ldots \ldots \ldots$

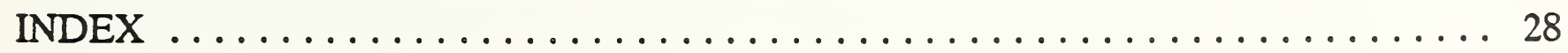




\section{Preface to the Second Edition}

As the semiconductor industry has grown in size, more and more people who are not semiconductor specialists have become involved with the industry. Some are clerical or other nontechnical persons, some are in other businesses such as venture capital, and some are in the communications media. In particular, the importance of the industry to the U.S. economy has introduced many economists and government people to the complexities of semiconductor technology which, at times, seem not to be well understood.

Yet some degree of understanding of the terms and concepts basic to semiconductors is often needed by those whose specialties are in other fields. This text, which is intended to meet that need, was originally prepared in 1976 to be part of $A$ Report on the U.S. Semiconductor Industry, by the Department of Commerce. That report was issued in September 1979. Both before the appearance of the Commerce report and since, there have been requests for this material from people who were first getting acquainted with semiconductors. Independent publication of the first edition of this report in early 1981 confirmed the belief that it would serve a continuing need. Demand continues to be modest but steady.

This second edition has been substantially revised and enlarged to bring it up to date. The industry moves rapidly and time has brought many changes in its technology. The subject is even more relevant to national economic well-being today. Suggestions from readers regarding possible improvements are welcome. 


\title{
SEMICONDUCTOR TECHNOLOGY FOR THE NON-TECHNOLOGIST
}

\author{
Robert I. Scace \\ National Institute of Standards and Technology \\ Gaithersburg, MD 20899
}

\begin{abstract}
The properties of semiconductor materials, the methods of processing them, and the solid-state products made from them are described in terms intended to be understandable by the lay person. The semiconductor industry has grown at a rate of 17 percent per year compounded for the last thirty years. Its products have declined in unit cost by a factor of 4.7 in current dollars (a factor of 18 in constant dollars) in the same period, irrespective of the vastly increased capabilities of today's products. This very satisfactory but anomalous behavior has attracted the interest of many who are not familiar with the technology of the industry, yet who need to have some understanding of it. This report is intended to help meet that need.
\end{abstract}

Key Words: Compound semiconductors; gallium arsenide; integrated circuits; semiconductors; semiconductor devices; semiconductor manufacturing equipment; semiconductor processes; semiconductor technology; silicon.

\section{INTRODUCTION}

This is a detailed discussion of semiconductor technology using nontechnical terms so readers not familiar with the subject can comprehend its complexity and breadth. While the knowledge underlying semiconductor technology comes primarily from the fields of physics and electrical engineering, the processes used to manufacture semiconductor devices rely heavily on chemistry, chemical engineering, and metallurgy. Design of semiconductor devices and operation of semiconductor factories both require computers and specialized software. A manufacturer of semiconductors will have on its professional staff ceramicists, chemists, computer specialists, electrical engineers, industrial engineers, mechanical engineers, metallurgists, physicists, and possibly specialists in optics. Because of the great diversity of technical information required to make semiconductor devices, no one kind of professional employee has sufficient skill to do the entire job. Semiconductor products result from the team efforts of a variety of professionals.

\section{BASIC MATERIALS}

Semiconductor materials generally are crystalline substances of relatively simple physical and chemical composition. Because of this simplicity, good theoretical understanding of the properties of semiconductors has been developed. The theory is extensively used to understand the behavior of solid-state devices and to design new ones. While there are many semiconducting compounds and elements, only a few have commercial significance. 
Originally, because of the relative simplicity of processing it, germanium was the material most commonly used. From the middle 1950 s to the middle 1960 s, silicon gradually became the primary semiconducting material for several reasons. First, since silicon is much more abundant than germanium, it is cheaper. (Sand is mainly silicon dioxide.) Second, germanium devices generally will not operate above $85^{\circ} \mathrm{C}$, whereas some silicon devices can operate up to $200^{\circ} \mathrm{C}$. Third, and probably most important, silicon has a tenacious, electrically insulating, protective oxide. The oxide of germanium is not protective. The properties of silicon dioxide have important consequences in the processing of semiconductor devices, and they have made possible the batch processing necessary to make solid-state devices at low cost. Therefore, the following discussion will deal mainly with silicon.

Compound semiconductor materials such as gallium arsenide are also becoming important for certain applications. Both gallium and arsenic are relatively scarce materials. For that reason, and because processing compound semiconductors is more complex than for silicon, compound semiconductor devices are and will remain more costly to produce. They are therefore used only in applications in which their performance is significantly better in some respect than that of silicon devices.

The crystalline structure of these materials is basic to their semiconducting action. As the word implies, semiconductors are neither good conductors of electricity nor good insulators but somewhere between. Silicon will be used for this discussion, but other semiconductors behave similarly. Pure silicon at room temperature has a very high electrical resistivity of $230,000 \mathrm{ohm}-$ centimeters, which makes it a poor conductor of electricity. This arises because the atoms in the silicon crystal structure are arranged so that each atom has four other silicon atoms around it at equal spacings. This so-called diamond cubic arrangement allows each of the four outer electrons of the silicon atom to be bound to the four neighboring atoms. In turn, the four neighboring atoms have their electrons similarly bound. Each interatomic bond in the crystal lattice therefore ties up two electrons, one from each of the atoms connected by that bond (called a "covalent" bond). Thus, all the outer electrons of the atoms in a perfect silicon crystal are bound together and are not available to conduct electricity, if the effects of temperature are ignored. At temperatures other than absolute zero, the atoms in a crystal vibrate about their nominal positions, and a few bonds may at any instant be disrupted. The electrons released this way do provide a slight amount of electrical conduction, and therefore the resistivity of pure silicon at room temperature is not infinitely high.

If, however, elements from neighboring columns of the periodic table are introduced into the crystal lattice, this arrangement of electrons is disturbed. For instance, if a phosphorus atom, having five outer electrons, is substituted for a silicon atom in the crystal, four of the five electrons will be bound to the crystal lattice, but one will be left to move freely within the crystal. This makes the crystal $n$-type, meaning that the principal carriers of the electric current are negative charges (electrons). Conversely, if a boron atom, having three outer electrons, is substituted for a silicon atom in the crystal, the electrons can complete only three of the four necessary bonds. The absence of an electron in the one remaining bond represents a departure from the symmetrical bond arrangement of a perfect crystal. It is possible for an electron from a neighboring bond to move into this space and create a vacancy, having a net positive charge, in the bond left behind. This vacancy, with its positive charge, can propagate through the crystal 
in a manner quite similar to that of an electron, with the exception that the sign is positive instead of negative. This charge-carrying entity is called a hole, and crystals in which this (positive) charge transport mechanism predominates are referred to as $p$-type.

The elements that cause $n$-type or $p$-type conduction are referred to as dopants or impurities. They are impurities only in the sense that they are not silicon. The silicon itself is quite pure as are the dopant elements. In fact, silicon is the purest raw material ever produced in tonnage quantities. With the exception of intentionally-added dopants, impurity levels of only a few parts per trillion are common in silicon used for semiconductor manufacture.

Dopant elements may be added to the silicon at several points in the semiconductor device manufacturing process. They can be added at the time that the single crystal of silicon is formed, or later by localized melting and recrystallization processes (alloying), by high-temperature diffusion processes, during the growth of additional layers of silicon, by high-energy bombardment (implantation), or by nuclear transmutation. As purchased, the doping of silicon is such that its resistivity usually falls between 0.001 and $1000 \mathrm{ohm}$-centimeters. Values of resistivity outside this range are achievable but not often used. The effects of dopant elements are quite significant, permitting silicon to have nearly any desired resistivity and either $n$-or $p$-type conductivity.

The boundary between a $p$-type region and an $n$-type region is called a $p$ - $n$ junction. The active portion of a semiconductor device consists of $n$ - and $p$-type regions and the junctions between them. The processes for making such devices provide for the introduction of dopant atoms in predetermined locations and amounts with precise control and reproducibility. The ways in which this is done will be discussed later.

Various external layers can be arranged to provide electrical insulation or electrically conducting connections to parts of the device. Of the insulators, silicon dioxide is most common partly because it is a product of some process steps and is there anyway, and partly because of its excellent electrical insulation properties. In some cases, layers of aluminum oxide or silicon nitride are used. Where insulation thicknesses of more than one micrometer (abbreviated $\mu \mathrm{m}$; see Appendix A for a discussion of these small, unfamiliar dimensions) are needed, various special glass formulations can be deposited on the wafer surface.

The most common metals used for connection to the silicon are aluminum and gold, since both are readily available in very high purity, can be vacuum-deposited easily, and form good electrical connections to silicon. Since a certain amount of silicon is dissolved by molten gold or aluminum in the process of making the electrical connections, a small amount of silicon is often added to the metal before it is deposited on the silicon to avoid excessive dissolution. Very complex sequences of metal layers may be used, though often at considerable extra cost. Heavily doped silicon, tungsten silicide, and other combinations of metal may be used. The metal films serve to connect the often minuscule regions of the semiconductor device with each other, and to provide regions to which external connecting leads can be attached.

In general, all materials used in a semiconductor device -- the silicon, its dopants, the coatings applied, and the metallization -- must be extremely pure. Because elements other than dopants often adversely affect the electrical properties of the device if they find their way into the silicon, 
they must be excluded. The only way this can be done is to use materials of the highest purity. Uncontrolled elements can lead to uncontrolled effects in processing and to uncontrolled device electrical characteristics at the end of the process. The same stringent demands of purity are made of the various chemicals used in cleaning, etching, and otherwise processing the silicon from beginning to end of the process. One of the major technical achievements towards making modern semiconductor devices possible, not widely known outside the industry, was the attaining of suitable purity levels in the wide range of ancillary materials needed for processing. When the semiconductor industry was in its infancy, such high-purity materials were known only in the research laboratory. Today, they are available off-the-shelf in most technically advanced parts of the world.

\section{BASIC DEVICES}

The $p-n$ junction diode and the transistor, both elementary devices, are useful in themselves and in combination with one another can make up integrated circuits.

Figure 1 illustrates a $p-n$ junction diode. Each of its two regions has a wire to connect it with the outside world. It is divided into two portions by the $p-n$ junction. In the upper portion, the majority carriers of electrical current are electrons (minus signs), while in the lower portion holes (plus signs) carry the current. If an external battery is connected such that the upper electrode (cathode) has a negative voltage applied and the lower (anode) a positive voltage, then the charge carriers in those regions are repelled from the electrodes and flow towards the $p-n$ junction, and in crossing that junction find themselves in regions of the device in which they are not in equilibrium. An electron, for example, flowing downward past the $p-n$ junction will combine with one of the holes with which it is surrounded and disappear. Other electrons will suffer the same fate. Conversely, holes flowing upward into the $n$-type region will combine with electrons

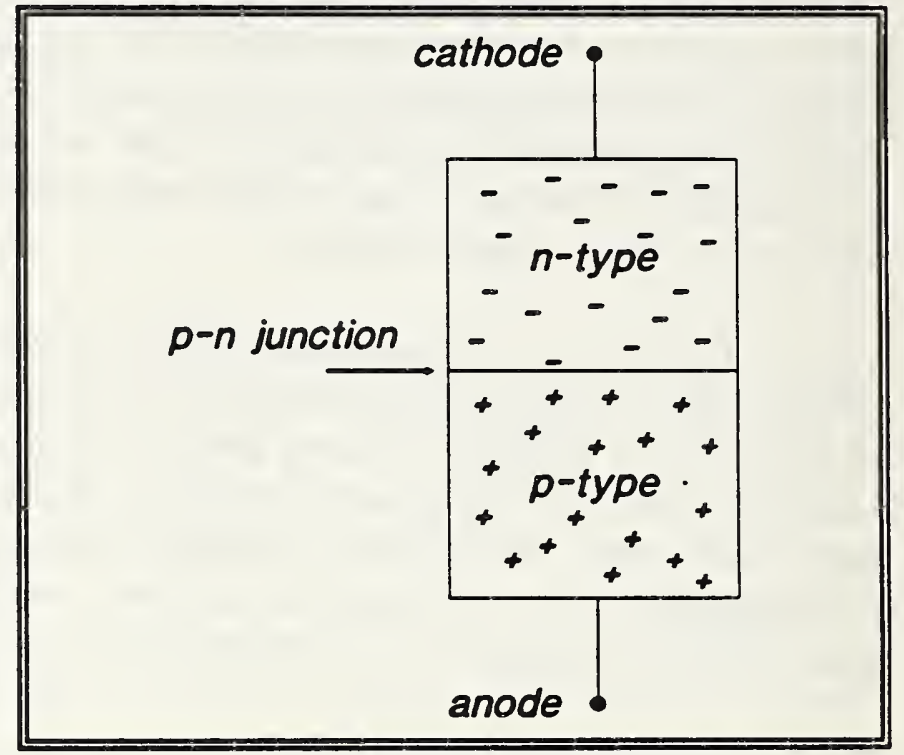

Figure 1. Elementary junction diode. there and disappear. This flow of electrons and holes across the $p$ - $n$ junction constitutes an electric current through the device and through the external circuit. This direction of polarity is called forward bias. If the upper electrode has a positive voltage applied and the lower electrode a negative voltage, the charges in the respective upper and lower regions are attracted towards the electrodes and away from the $p-n$ junction leaving behind a region with no free electric charge carriers. This region acts effectively as an insulator, and virtually no current exists. This direction of polarity is referred to as reverse bias. This diode or rectifier then acts as an electrical valve that permits the passage of current in one direction and obstructs current in the opposite direction. 
Figure 2 is a schematic sketch of an $n p n$ (bipolar junction) transistor. (There are other kinds of transistors, too.) The upper $n$-region is biased rather strongly positively by the external battery on the right of the figure, and the upper $p$ $n$ junction is therefore reverse biased. There is very little current in the circuit connecting the upper $n$-region with the lower $n$-region. But when a positive voltage is applied to the central $p$-region by the battery on the left, holes pass from the $p$ region into the lower $n$-region and electrons pass from the lower $n$-region into the central $p$-region. A current therefore exists in the left-hand loop of the sketch. Although the figure does not show it, the $p$-region in a real transistor is extremely thin. Therefore, most of the electrons passing from the lower $n$-region into the central $p$-region do not have time to recombine with holes there, but they survive to reach the upper $p-n$ junction. At this point they are attracted to the much larger positive voltage on

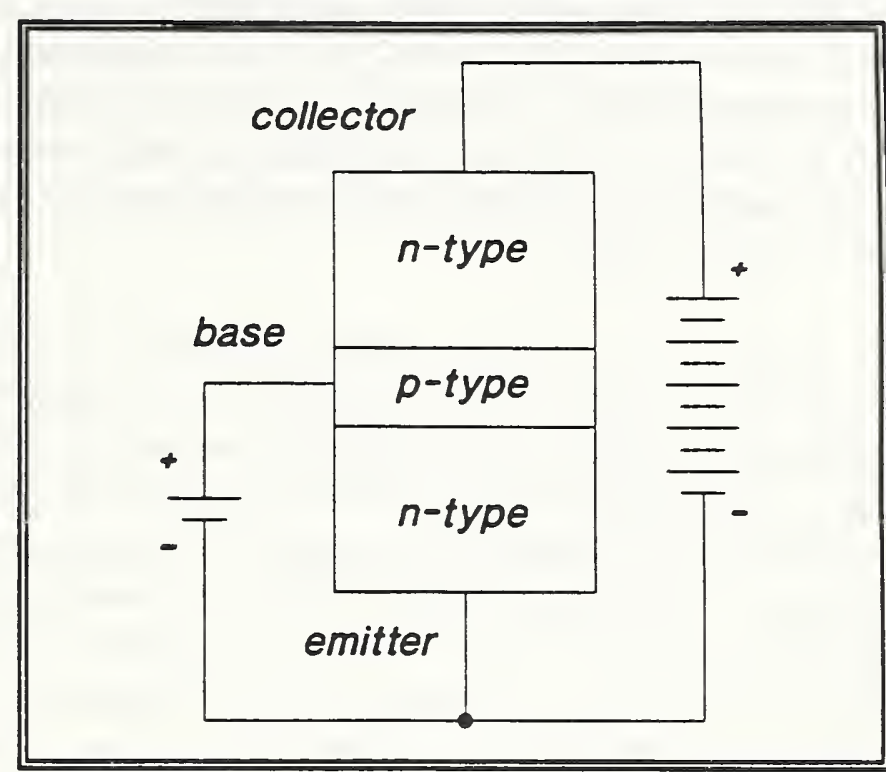

Figure 2. Elementary $n p n$ transistor. the other side of the junction and are swept across it. This constitutes a current in the righthand loop of the sketch. If current in the left-hand loop is interrupted by breaking the circuit (by disconnecting the battery on the left, the current in the right loop ceases. Similarly, an increase in current in the left-hand loop will lead to an increased current in the right-hand loop. Thus one current controls another; this is the essential property of the transistor. Transistors amplify because a small change in current in the left-hand loop results in a large change in current in the right loop. The device illustrated is a junction transistor, so called because it contains two $p$ - $n$ junctions. Other transistors, such as the junction field-effect transistor and the metal-oxide-semiconductor field-effect transistor (MOSFET), accomplish a similar function although by different means.

A schematic illustration of a MOSFET is shown in Figure 3. This example is an $n$-channel MOSFET, so named because the carriers of current in the device are electrons. The figure shows a piece of $p$-type silicon with two $n$-type regions formed in its upper surface. Most of the top surface is covered with a layer of silicon dioxide, shown without shading. Metal layers (the diagonally-shaded regions) are formed on the top surface. Two of these connect through holes in the silicon dioxide to the two $n$-type regions, the source and the drain. The third metal layer, the gate, does not connect to the silicon at all, but is insulated from it by a thin layer of silicon dioxide.

The channel (shown by the arrow) is not present as a permanent feature of the device, but is created by applying suitable electrical voltages to the device's terminals. Four electrical connections are shown, but usually the substrate and the source are connected together. If the gate terminal is not connected to an external voltage, the source/substrate has a negative voltage 
applied, and the drain has a positive voltage applied, no current will flow between the source and drain. The drain-substrate junction is reverse biased under these conditions. But if, in addition, the gate has a positive voltage applied to it, electrons will be attracted to the surface region of the substrate directly beneath the gate electrode. The holes normally present in the $p$-type substrate material are simultaneously repelled by the positive gate voltage. The net effect is to create a thin $n$-type region just below the gate electrode, forming the channel. The channel is a path having $n$-type conductivity which connects the source to the drain and allows current to flow between them.

The degree of conduction of the channel is directly related to the amount of positive electrical voltage on the gate, so the MOSFET can operate as an amplifying device just as the junction transistor can. The principal difference between the two types is that the junction transistor is a current-controlled device, while the MOS transistor is a voltage-controlled device. The layer of silicon dioxide beneath the gate prevents any current from flowing between the gate and the substrate. Some applications are better served by one kind of transistor rather than another. Just as there are two types of junction transistors, $n p n$ and $p n p$, having complementary electrical properties, so there are two types of MOS transistors, $n$-channel and $p$-channel, the latter being of the same construction as illustrated in Figure 3 but with $n$ - and $p$-regions, and the polarity of the applied voltages, interchanged.

The device behavior described above depends upon the flow of electrons and holes under the influence of external currents and voltages. While the operation of other semiconductor devices will not be discussed in detail, it should be kept in mind that all devices, from the simplest $p-n$ diode to the most complex integrated circuit, operate using elementary concepts such as these. The art of making semiconductor devices is simply that of arranging the $n$-type and $p$ type regions, the junctions between them, and their interconnections so that useful electrical properties result.

\section{SEMICONDUCTOR MANUFACTUR- ING EQUIPMENT}

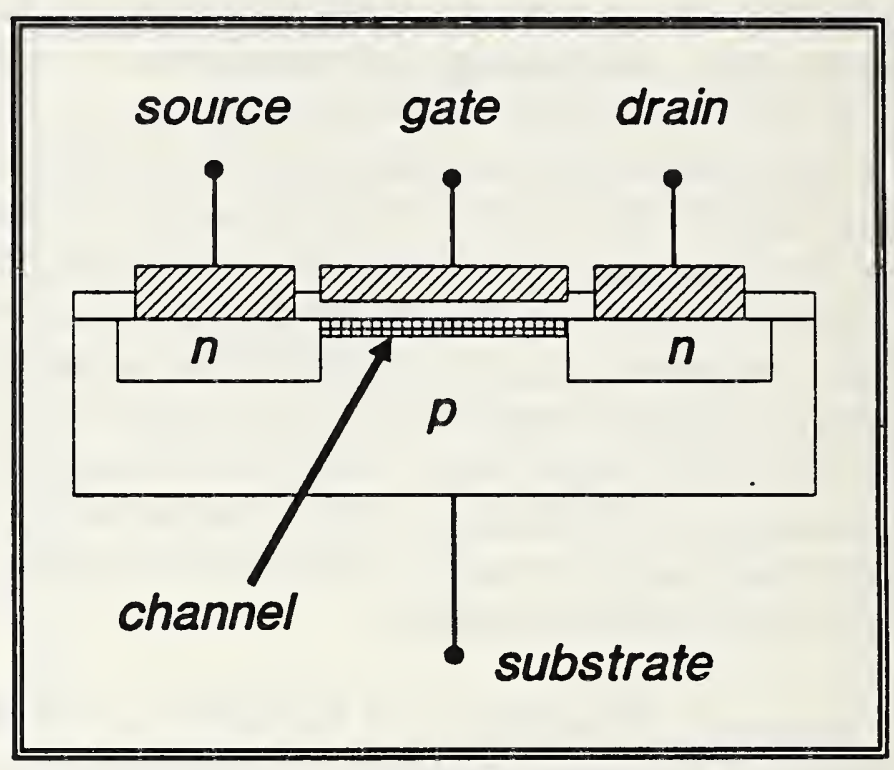

Figure 3. n-channel MOS field-effect transistor.

Much of the capital equipment used in semiconductor processing originally evolved from laboratory tools. The extreme demands of cleanliness, precise dimensional control, or uniformity of temperature, unique to the semiconductor industry, have led to the creation of supporting industries manufacturing highly precise equipment for use in making semiconductor devices and for characterizing materials and manufacturing processes. Equipment companies work closely with their customers to understand the requirements of the processes to be executed by the equipment. Today, this equipment is highly specialized, computer-controlled, and expensive. Much of the process technology described below is embodied in the equipment. 


\section{PROCESSING TECHNOLOGY}

Many techniques are used to manipulate the dopants and other parts of the semiconductor device structure to achieve useful results. The manufacture of a given kind of device may use only some of these processes, but they will all be briefly described to illustrate the wide range of technical concepts necessary to make semiconductor devices. Any semiconductor manufacturing plant must be able to perform these processes in a controlled and reproducible fashion if it is to make devices with any degree of success.

\subsection{Crustal Growth}

Most of the metallic and ceramic objects with which we are familiar are composed of many tiny crystals of the material in question and are described as polycrystalline. Generally, these small crystals can be seen only under high-power microscopes. In contrast, semiconductor devices are manufactured using a starting material that is a large single crystal. Monocrystallinity is required because the boundaries between crystallites in ordinary materials have different physical properties from the body of the crystal. If one attempted to make semiconductor devices from polycrystalline silicon, the diffusion processes and other techniques used to introduce the dopants into the silicon would result in dopant concentrations at the crystallite boundaries different from the concentrations in the interior of the crystallites, and the desired degree of control over the structure would be impossible.

Semiconductor-grade silicon is produced in a chemical factory. (See Appendix B for a description of this process.) Most manufacturers of semiconductor silicon are also substantial manufacturers of other chemicals. One of the intermediate materials in silicone $e^{1}$ manufacture is particularly suited for the preparation of the semiconductor-grade silicon. This is reduced to polycrystalline high-purity silicon in the form of long slender rods. These rods are converted into single-crystal silicon of the appropriate doping and physical dimensions in one of two ways.

In the Czochralski method, a quantity of silicon is melted in a quartz crucible under an atmosphere free from oxygen. A slender single-crystal bar (seed) of silicon is introduced into the melt from above and a portion of the melt begins to freeze onto the seed. If the temperature and other conditions are correct, the growth onto the seed crystal is a single crystal whose crystal structure is a continuation of that of the seed crystal. In this fashion, the liquid silicon in the melt is converted into a cylindrical ingot of silicon without any intercrystalline boundaries. The doping of a Czochralski crystal is performed by adding appropriate dopant elements to the melt prior to growth. In commercial practice, crystals up to 200 millimeters (mm) (8 inches) in diameter and over 1 meter (40 inches) long are available. Larger crystals can be made.

While the Czochralski process produces semiconductor silicon appropriate for manufacture of many kinds of devices, the silicon contains an appreciable amount of oxygen dissolved from the

1 Distinct from silicon. Silicones are a class of chemicals similar to organic chemicals, but with a silicon-oxygen chain as a structural basis rather than the carbon-carbon chain of organic chemicals. 
quartz crucible in which the melt was held. In addition, during the growth process some segregation of the doping element takes place. The solubility of most dopants is greater in the melt than it is in the crystal. The concentration of dopant in the melt thus increases continuously during the growing process. The finished single crystal does not contain a uniform concentration of the doping element from one end to the other and contains oxygen that is troublesome in the manufacture of some devices, especially those required to operate at high voltages, but beneficial in making other devices. Oxygen tends to make wafers less subject to warping at high temperatures and, with the proper heat treatment, can form localized centers (precipitates) which attract and hold ("getter") undesired impurities which may get into the silicon as contaminants during its processing.

Some of these difficulties can be overcome by growing silicon crystals by the float-zone method. In this technique, a cylindrical rod of polycrystalline silicon is suspended in a chamber in an atmosphere free from oxygen. The bottom of the rod is melted with a high-frequency induction coil, forming a liquid drop hanging from the bottom of the silicon rod. A seed crystal is introduced into this silicon drop from below, and by controlling the temperature of the molten region and the other thermal parameters of the apparatus, silicon is made to grow upon the seed as a single crystal. By slowly raising the induction heating coil, the molten region of the bar is also raised, dissolving silicon from the polycrystalline rod above and growing it as single crystal onto the crystal ingot supported by the seed below. The molten region is held in place by a combination of surface tension and magnetic forces from the induction heating coil. Doping in this process is either by means of a small quantity of concentrated dopant added to the initial melted drop before growth on the seed has begun, or by incorporating a gaseous compound of the dopant into the atmosphere around the growing crystal in a concentration such that it dissolves in the molten region at the same rate at which dopant atoms are withdrawn from the molten region by the growing single crystal. Float-zone silicon of 100 -mm (4-inch) diameter is currently commiercially available. This material is quite free from oxygen and can have a much more uniform distribution of doping species incorporated in it in comparison with Czochralski silicon.

A technique for producing exceptionally uniformly doped $n$-type crystals of silicon employs neutron irradiation. If an undoped crystal is exposed to neutrons in a nuclear reactor, some of the silicon atoms will absorb a neutron and be transmuted into phosphorus atoms. Since this occurs at random, the newly created phosphorus (an $n$-type dopant) is distributed uniformly throughout the crystal. Such a degree of doping uniformity is impossible to obtain by the methods described earlier. Local fluctuations in resistivity caused by nonuniform conventional doping may amount to 20 percent of the average. Since resistivity is one of the principal factors influencing the maximum blocking voltage of a $p-n$ junction, these deviations are one cause of variations in the voltage ratings of devices made from conventionally doped silicon. This is a particular problem in large, high-voltage devices used for power control. Because of the large area of the silicon wafers from which these devices are made, there is a high probability that there will be a lower than average resistivity region somewhere in the wafer. This causes the breakdown voltage at that point to be less than at other places, thus limiting the voltage rating of the device to a lower value than it otherwise would be. The uniform resistivity of neutronirradiated silicon allows this difficulty to be avoided. Devices made from this material can have a 15- to 20-percent higher maximum voltage rating without the necessity for making any changes 
in design and without affecting any of the design trade-offs that would otherwise have to be made.

\subsection{Mechanical Processes}

Crystals obtained by either the Czochralski or float-zone method are usually ground to a predetermined diameter with grinding equipment using diamond abrasives. Since uniform diameter of the crystal is necessary for the silicon to fit into handling fixtures and process equipment, the industry has standardized on a few fixed diameters for silicon wafers. At this point, the silicon has a market value ranging from 50 cents to $\$ 1$ per gram ( $\$ 230$ to $\$ 460$ per pound). Because of this high value, subsequent operations must be conducted so as to minimize the waste of silicon.

The crystal next is sawed into thin wafers, using diamond-edged blades. The difficulty of this operation can be appreciated when one considers that silicon is as brittle as glass, and the wafers may be as thin as $0.25 \mathrm{~mm}$. Large-diameter thin wafers are delicate, and the sawing operation must be conducted with great care. For many applications the wafers need not be so thin, and for reasons of mechanical strength are more often in the thickness range of 0.5 to $0.8 \mathrm{~mm}$. Wafers are etched and lapped on both sides to remove the structural damage done to the surface layers of the wafer during the sawing operation. The edge of each wafer is ground to round the corners where the two faces meet the edge, to reduce the likelihood of chipping off bits of silicon. If this occurs, the wafer is strained and is more likely to break during processing. Worse, silicon chips are a source of contamination during processing. For most transistor and integrated circuit manufacture, the wafers are then polished to a mirror finish on one side. In this way all mechanical damage to the crystal on that side is removed, preparatory to further processing. In addition, the polished face must be extremely flat to allow successful imaging of the fine details of an integrated circuit.

It is necessary at several of the preceding points to clean the wafers thoroughly. Mechanical operations are usually relatively dirty. The dirt includes small particles of silicon as well as other materials. These must all be removed from the silicon wafer in preparation for later work. Cleaning involves solvent degreasing, heated detergent solutions under ultrasonic agitation, and physical wiping operations.

\subsection{Epitaxy}

The cleaned, polished wafer is now ready for growth of a layer of silicon by an epitaxial process, which is necessary for some types of products. Epitaxy denotes a crystalline growth such that the atomic arrangement on the surface of the original wafer (substrate) is continued into the growing layer. Growth is done at high temperature in an atmosphere containing a silicon compound that decomposes on the hot wafer and leaves silicon there. A dopant compound is also present, which decomposes in a similar way to provide the desired doping of the growing silicon layer.

Generally, an epitaxial layer is from 2 to $20 \mu \mathrm{m}$ thick and is the silicon layer in which the final devices will be formed. The function of the substrate is simply as a host surface for the epitaxial layer and as a thick mechanical support. The use of epitaxy permits the manufacturer to buy 
silicon using relatively undemanding specifications (thus keeping the cost down), and to grow a layer tailor-made for the specific kind of product to be made. It also prevents certain electrical problems in complementary metal-oxide-semiconductor (CMOS) types of integrated circuits.

\subsection{Oxide preparation}

A film of silicon dioxide may be required as a preliminary to a diffusion or implant operation or to cover layers previously placed on the surface. Thermal oxidation is a simple way to grow an oxide layer over the entire surface of a wafer. The oxidation takes place in a tube furnace at a precisely maintained temperature, usually between 900 to $1200^{\circ} \mathrm{C}$, in a controlled atmosphere containing oxygen. Not only are the composition of the atmosphere and its purity maintained, but the temperature is also closely controlled. Typical present-day furnaces for semiconductor manufacture can maintain temperatures of $1000^{\circ} \mathrm{C}$ with a precision of one-half degree over a space exceeding 1 meter in length and $200 \mathrm{~mm}$ in diameter. Oxides are grown from a few thousandths of a micrometer to one or two micrometers thick, in times ranging from a few minutes to many hours, depending on thickness and furnace temperature.

Alternatively, oxides (and other films) may be deposited on the wafer by several means including chemical vapor deposition or vacuum sputtering. The choice of method depends on the electrical properties required of the oxide and on the effects that process time and temperature will have on what has been done to the wafer previously. The features of integrated circuits are exceedingly small. Too much exposure to high temperatures will cause diffusion of precisely placed dopants, for example, to places where they should not be, thus blurring the patterns. Preference is usually given to processes which can be done at low temperatures rather than high ones for this reason.

\subsection{Micropatterning}

To form patterns on a silicon wafer, a photographic. process of great precision is used. A thin metal layer on a glass or quartz plate (the photomask, or simply, mask) contains an image of the desired pattern. A layer of photosensitive material (resist) is spread on the surface of the silicon wafer and dried. It is exposed to ultraviolet light through the mask to cause chemical changes in certain areas of the resist. The pattern is developed chemically, leaving areas of the wafer covered with resist while other areas are clear.

For a diffusion or implantation process, the wafer may then be etched to remove silicon dioxide in the clear areas, following which the remaining resist is stripped away. Doping during diffusion or implantation reaches the silicon only through the windows in the oxide, but not elsewhere. Or, for implantation or for metal deposition processes, the resist may be left in place. After the following process, the resist is stripped off, leaving a pattern of doping from the implant or a pattern of metal from the metal deposition. For room-temperature processes, the resist is often a sufficient barrier, but in high-temperature processes the resist would be destroyed.

The mask containing the pattern that was used in the preceding step is generated itself by a complex sequence of operations. During the design process (see section 8), a computer creates a data base which contains pattern information for each layer in the device. This data base is 
sent to the mask-making facility, which may either be internal in larger companies or an outside supplier.

One of two mask-making processes is used. For moderately small patterns, down to about 10 $\mu \mathrm{m}$ in minimum dimension, an optical pattern generator may be used. This machine exposes the pattern, point by point, on a resist-coated metallized mask blank. After the resist is developed, the metal is etched to create the pattern in a master mask. For features of smaller size, the pattern generator cannot form a sufficiently precise image, so an electron-beam machine is used. (The electron-beam process can be used for large patterns as well.) Electrons can form precise images at a much smaller scale than can optical equipment, but the electron-beam equipment is substantially more expensive. The electron-beam machine writes the desired pattern on the resist much like a television set writes pictures on its screen, point by point but quite rapidly. Once the pattern is written, the resist and metal are processed in the same way as for the optical pattern generator case.

Most integrated circuit processes require a set of ten to twenty masks for the sequential steps necessary to fabricate the device. The images on the masks can be the same size as the final circuit, or it can be a few times larger. In the latter case, the final size image is produced on the silicon wafer by optical reduction through a projection lens at the time the silicon is being processed.

In repeating photomasking operations, it is necessary to align the new pattern precisely with that already on the wafer. This is done with the aid of a sophisticated mechanical apparatus called a photomask aligner, of which there are several types. In making complex integrated circuits, it is necessary to align patterns to within typically 10 percent of the minimum feature size. Difficulties in this process will be appreciated when it is realized that pattern features as small as $0.8 \mu \mathrm{m}$ are used in making integrated circuits. A complex integrated circuit may contain millions of features of these dimensions. The patterns in sequential masks in a set thus must align with one another to within substantially less than that dimension, such that all the patterns in the sequence will be correctly aligned.

Further, any defects in the images will be reproduced on the silicon wafer as defects in the pattern of resist. This leads frequently to failure of that particular segment of the wafer to produce a good final device. Scratches, dirt, and misalignment all contribute to losses of what would otherwise be good devices. Such defects in pattern definition are a major cause of yield loss in device manufacture. In large, complex integrated circuits, the yield may be as low as 5 percent in early manufacturing. That is, only one good circuit is produced for every 20 that are started at the beginning of processing of the silicon wafer. As experience is gained and process control improves, the yield usually rises to over 90 percent for a well-controlled process.

Older circuits had larger minimum feature sizes, and masks and aligners were made to expose the entire wafer at one time using "contact" printers, in which the mask and wafer were in or nearly in contact. Masks were easily damaged in this process, so more advanced optical aligners used a projection method. Projection printing allowed the mask to be better protected. Difficulties in achieving sharp focus (partly for optical reasons and partly because wafers are not always sufficiently flat) and exact alignment over the entire wafer led to development of aligners 
which expose only one chip or a small block of chips at a time. These machines step from one location to the next, exposing each in sequence, and thus are called wafer steppers. To reduce the incidence of defects in the patterns on the silicon, masks for projection printers or steppers are rigorously inspected, and defects are repaired before the masks are used. This is a costly procedure, but the long life of masks in this equipment and the reduction of yield loss makes it worthwhile.

There are serious optical and measurement problems related to the photomasking process. The reduction lenses in steppers are specially designed for this purpose and are very expensive. Such lenses must be capable of forming an image without distortion and with excellent edge definition in a very flat focal plane. On the photomask the dimensions are so small that accurate measurement is difficult. Lines on these masks are only $1 \mu \mathrm{m}$ wide (only 2 wavelengths of green light) for printing at unity reduction ratio. They do not appear well defined in measuring microscopes because their size is so close to the dimensions of the light waves used to observe them. The problem of verifying that each of the millions of lines and spaces on a photomask is of the proper size and in the right place is staggering.

Under some circumstances, electron beams or x-rays may be used to create patterns. The same kind of electron-beam writing machine that is used for making photomasks can be used to write patterns on wafers. There is an economic problem, however. The machine costs twice as much as a wafer stepper and processes fewer than a third as many wafers in an hour. The method is used either for exceedingly finely detailed patterns $(0.5 \mu \mathrm{m}$ feature size or smaller $)$ or for processing wafers when only a few of them having a given pattern ever will be needed. This is often the case for application-specific integrated circuits.

The trend toward denser circuits means that more transistors having smaller feature sizes must be produced on a single chip. The alternative is making the chips larger, which means that fewer can fit on a wafer. Larger chips are also more likely to include a defect caused by random bits of dirt falling on the wafer. Both of these factors make the chips more expensive. But to make finer details, one must expose the patterns with shorter wavelengths of light. This has been a continuing trend for a decade, but the possibilities of continuing in this direction are becoming limited. Good high-intensity sources of short-wave ultraviolet light at the necessary wavelength are not conveniently available. Certain types of lasers can be used but they are expensive and still quite experimental in nature. Even if a good light source is found, at such short wavelengths most optical glasses available for making projection lenses for steppers are no longer transparent.

For these reasons, $\mathrm{x}$-ray light sources are being seriously considered. $\mathrm{X}$-rays are electromagnetic radiation, as light is, but of a much shorter wavelength. The use of $\mathrm{x}$-rays presents further problems which must be overcome. Conventional $x$-ray sources, such as those used for medical purposes, produce radiation which spreads from the source in all directions. Means to collect and focus these rays in one direction are only beginning to be developed. If this is not done, the brightness of the $\mathrm{x}$-ray source is not sufficient to expose the photoresist on a wafer in a short enough time to give a useful production rate.

One approach uses an electron synchrotron (particle accelerator), originally developed as a nuclear physics research tool, to produce $\mathrm{x}$-rays of the right wavelength. These machines 
produce extremely intense $x$-rays which come off the machine in straight, parallel beams. This is just what is desired for making chips, but synchrotrons are very expensive light sources. One such machine can supply $\mathrm{x}$-rays to as many as about 15 exposure stations, but the installation will cost at least $\$ 25$ million, not including the cost of the building to enclose it. Device makers are concerned that the failure of the synchrotron would shut down an entire factory. In addition, much work remains to be done to develop suitable photoresists and masks. Many experts consider the mask-making problem to be at least as difficult as the engineering and development of commercially useful synchrotrons.

At present, it is difficult to predict which of these (or other) alternatives will prove to be the most suitable. The one certainty is that producing chips with feature sizes of $0.25 \mu \mathrm{m}$ or less will require extremely costly tools.

\subsection{Diffusion}

Following the creation of open areas in the oxide coating, and the removal of the photoresist, wafers can be diffused with a dopant. This is a batch process, in which a number of wafers are inserted in a furnace at a precise temperature, usually between 900 and $1200^{\circ} \mathrm{C}$, in a closely controlled atmosphere. The atmosphere contains a quantity of the dopant to be diffused into the silicon surface to dope the regions that are free from oxide. The concentration of the dopant in the silicon is related directly to its concentration in the atmosphere, and the depth to which it penetrates is related to the square root of the time that the wafers are exposed to the atmosphere in the furnace and of the temperature. Diffusion times may range from a few seconds to many hours depending on the depth of diffused layer to be produced. High purity sources of diffusing atoms may be either gaseous, liquid, or solid.

\subsection{Ion Implantation}

Ion implantation permits the placement of dopant atoms into the wafer at or near room temperature, rather than at the high temperatures required in diffusion. Integrated circuits having minimum feature sizes of around $2 \mu \mathrm{m}$ or less must be processed with a minimum amount of exposure to high temperatures. Any high-temperature process, whether in a diffusion furnace or anywhere else, causes diffusion of dopants and other impurities to occur. If this diffusion is allowed to proceed, the fine features become blurred and can even merge with one another. Today's process sequences also involve many more steps than in earlier times. Both of these facts make the use of low-temperature processes essential.

In ion implantation, a high-energy stream of dopant atoms is directed toward the silicon wafer in a high vacuum. The atoms penetrate into the surface of the target wafer to a depth depending upon their kinetic energy. The number of atoms implanted in a given volume of the target wafer can be very precisely controlled, as can the depth of penetration. Furthermore, distributions of dopant atoms not possible to achieve by other means can be produced by ion implantation. This technique is now routinely used in the manufacture of high-performance integrated circuits. 
Implantation causes severe dislocation of the atoms near the surface of the wafer. This damage must be removed by a brief exposure to high temperatures (annealing). Formerly this was done in a diffusion furnace. Now it is more common to use rapid thermal annealing equipment which raises one wafer at a time quickly to a high temperature, perhaps $1000^{\circ} \mathrm{C}$, for only a few seconds and rapidly cools the wafer again. This is sufficient time for the surface atoms to rearrange themselves without causing significant amounts of diffusion.

For most products it is necessary to repeat the preceding steps of oxidation, photomasking, and diffusion or implantation a number of times to create the desired structure. Simple transistors require two or more diffusion steps plus a masking operation to expose the surface regions where electrical contact is required. After the metal connecting layer is deposited as described below, a photomasking and etching operation delineates the desired pattern of metal. Modern integrated circuits may require twenty or more separate photomasking steps and have as many as four separate layers of conducting materials on their surface to interconnect the individual devices that comprise the chip.

\subsection{Passivation}

Frequently it is necessary to provide an inorganic coating on the semiconductor wafer to protect it from exterior influences. Such layers are called passivation layers, and they range from a $1-\mu \mathrm{m}$-thick layer of silicon dioxide to relatively thick deposits of special glasses. The objective is to render the semiconductor chip inert to attack by external chemical species. In former times this protection was provided by a hermetic enclosure. Today, however, most devices are packaged in less expensive nonhermetic assemblies. It is necessary to arrange for the protection of the chip in spite of the nonhermeticity of the package. One can regard passivation as a technique for shrinking the hermetic package down directly onto the surface of the semiconductor chip. Particularly in power devices, passivation is a necessary step to provide the low surface leakage necessary for high-voltage junctions. Many power device processes leave the intersection of the $p-n$ junction and the surface of the device unprotected by any material. Considering the fact that the dielectric breakdown strength within a $p-n$ junction may be as high as 200,000 volts per centimeter, and that most gases, including air, break down at around 2000 volts per centimeter, the need for passivation of the edge of the $p-n$ junction becomes obvious.

Regardless of the kind of device, a $p$ - $n$ junction exposed to the outside world will not have stable reverse-biased electrical characteristics. In fact, many $p-n$ junctions that are protected by a thin silicon dioxide layer are still not stable over long periods of time due to diffusion of mobile ions through the silicon dioxide overcoat. Passivation of the device requires the isolation of the junction from these influences by the addition of inert surface layers. For low-power devices such as integrated circuits, silicon dioxide, silicon nitride, and aluminum oxide have been used with considerable success in protecting oxide-coated junctions from diffusion of external species. Higher voltage devices generally employ thicker coatings of glass applied in a variety of ways.

\subsection{Metallization}

Silicon is not a metal. It is not possible to make electrical connection to silicon in the ways commonly used to make connections to metals. Yet external connections are required, and are 
usually provided by the evaporation in vacuum of thin metal films onto selected areas of the surface of the semiconductor chip. In discrete devices, such as individual transistors, these provide metallic surfaces to which external metal leads may be attached. In integrated circuits, these evaporated layers also provide the interconnections between regions of the surface that need to be connected together electrically. In many cases these interconnections never are connected to the outside world at all. Various metals and multimetal systems are used for these purposes. Nearly every metal in the periodic table has been used at some time in a production semiconductor device for making electrical connection to the silicon. However, the most common are aluminum, polycrystalline silicon, and multilayer systems involving platinum, titanium, chromium, nickel, silver, or copper.

\subsection{Testing}

Once the processing of the wafer has reached a point at which metal contact regions are available, the devices can be electrically tested to some extent. It is not possible to carry out the full range of electrical tests, because the device is not yet mounted in a package that permits removal of substantial amounts of heat. However, low-power characteristics can be measured on discrete devices and the functioning of most integrated circuits can be checked. Testing at this stage is one of the most valuable tools in controlling cost, since defective parts can be weeded out and discarded without incurring further cost in their processing. At this point, a wafer of silicon will typically contain a very large number of apparently identical semiconductor devices. The testing apparatus has an array of precisely positioned, needle-like probes, which are arranged to descend on the contact pads that make electrical connections to each individual device. In the interests of speed and accuracy, the test is conducted by computer-controlled equipment. When a defective device is detected, it is often marked with a dot of identifying ink. This permits that particular device to be discarded after the wafer has been separated into individual parts. Alternatively, the test equipment records the location of defective devices. This information is used later when the individual devices are selected from the wafer during the assembly process.

\subsection{Dicing}

The separation of individual dice ${ }^{2}$ from the wafer may be accomplished by either scribing the surface of the wafer with a diamond-pointed tool, or by engraving the surface in the same pattern by the use of a high-powered laser beam, and subsequently cracking the wafer along these marks. Both operations are referred to as scribing, the diamond-point-scribing process being the older. Laser scribing provides improved control of the breaking process that follows. Alternatively, the dice may be cut apart with a thin diamond-edged saw blade. In many cases, this scribing operation is performed at the plant location in which previous processing has been carried out, but the wafers are not broken until they are delivered to the plant where the dice are to be assembled into completely packaged devices, which may be halfway around the world. In this way the operations requiring the highest degrees of skill and equipment investment are

2 Dice, chips, and pellets are the same. These terms refer to a segment of the wafer which contains usually one device or integrated circuit and which will be assembled into a package. 
confined to the processing plant, while operations requiring lesser skills are at the assembly location.

\section{ASSEMBLY}

The regularity of the pattern of dice in each wafer is valuable to the assembler of devices. If the wafer is broken and the dice become displaced from their initial positions, this regularity is lost. At assembly, each die must be identified, oriented, and put in the proper position for further assembly. This costs money. If the position and orientation of each die can be maintained, cost can be saved. This can be done by mounting a scribed wafer on a thin plastic membrane, separating the individual dice, and then stretching the membrane uniformly in two directions. The individual dice follow the stretched membrane and retain their relative position and orientation. During subsequent assembly operations, the devices can be picked from the membrane and positioned with a minimum of additional effort and cost.

The assembly of transistors and integrated circuits into finished devices generally involves mounting each individual die into a package. Often a metallic bond is created, using a layer of gold between the bottom of the silicon chip and the metal surface of the package. The package and chip are raised in temperature until the gold fuses with the silicon and forms a strong bond. Electrically conducting epoxy cements are also often used.

In devices that must dissipate a significant amount of power, other techniques are employed. Generally, the part to which the device is to be attached is made of copper whose high thermal conductivity is useful in extracting heat from the semiconductor chip. Bonding of this type of semiconductor device is done using a variety of metallurgical methods, but rarely with an epoxy or other material with poor thermal conductivity.

In very large, high-power, devices, the silicon may be bonded between two pieces of a metal such as tungsten. Tungsten has a thermal expansion coefficient similar to that of silicon and has good thermal and electrical conductivity. Its great stiffness provides a rigid backup for the silicon, protecting it from external mechanical forces. In hockey-puck shaped packages, this tungstensilicon-tungsten sandwich is assembled between copper end plates, but not bonded to them. The flexible diaphragm-like end plates of the package then permit the copper surfaces to be clamped by external pressure to the silicon-tungsten sandwich to provide good electrical and thermal paths to the device. The absence of a solid metallic bond permits differential expansion to take place between the tungsten and the copper, which have differing thermal expansion coefficients, without the risk of deforming a metallic joint and consequent fatigue failure after many heating and cooling cycles.

In low-power devices, once the die is mounted in the package, it is necessary to connect the individual metal contact patterns to the external connections of the package. The usual approach employs fine gold wire and the thermocompression bond technique. In this procedure, the end of the gold wire is melted into a spherical droplet, which is then solidified before being pressed against a metallic area on the silicon chip. A combination of temperature not as high as the melting point and high physical pressure serves to weld the wire ball to the metallization on the surface of the silicon. The wire is then led from this point to the external package 
connection where it is welded in a similar fashion. These wires range in diameter from 25 to 150 $\mu \mathrm{m}$. A complex integrated circuit may require several hundred such connections to be made between the chip and the package.

Once the electrical connections are complete, it is necessary to enclose the device to protect it mechanically from the shocks of the external environment. Silicon is brittle and will not tolerate physical abuse. Therefore, the package in which it is contained must protect it. In many cases the packages are molded of plastic materials, while in other cases the package is a metal and ceramic assembly. Semiconductor dice are also sold without any packaging at all. These are used by sophisticated customers who mount and connect these devices to other circuits of their own manufacture. With reasonable care it is possible to test and ship unpackaged dice to another location in large quantities without damage.

\subsection{Final Testing}

Final electrical testing of semiconductor devices is done by computer-controlled equipment. The number and complexity of tests are large, and the precision with which they must be conducted is high. Furthermore, given the large amount of data obtained during a test sequence on a single integrated circuit and the complex logic that governs decisions regarding the fate of the device under test, manufacturers must use computers for control of the test sequence.

In most cases it is not possible for a semiconductor process line to deliver a part that is characterized by a single product type number. The number of serial steps in the process and the inevitable variability of the product mean that the output distribution from a product line will be wide enough that it must be subclassified into parts having different electrical ratings. This sorting may be based on electrical characteristics, response of the device to temperature extremes, or other factors. Devices with a high degree of uniformity and that are operable over wide temperature ranges command premium prices. Other devices, perhaps made on the identical line at the same time, which have less desirable characteristics but which still function adequately, are sold for lesser prices. Additional sampling tests are done to explore the performance of the device in high stress environments or over long periods of time, to assure stability and long life. The testing costs incurred in qualifying devices for very high reliability service, such as in cardiac pacemakers or in satellites, may exceed the cost of manufacturing the device many times over.

\section{PROCESS CONTROL}

The subject of process control deserves separate discussion. The reader can appreciate from the foregoing description that semiconductor device manufacture is a long and delicate chain of events with more opportunity for failure than for success. Disaster in the form of catastrophically low yield is always lurking nearby, and the process engineer is the person who must control the many variables and keep the factory running.

Each of the processes described above produces a change in the silicon wafer, be it a pattern in photoresist, a diffused layer, or a deposit of contact metal. The majority of such changes are irreversible. If the process has been done incorrectly, expensive scrap has been created. 
The key to control is measurement. Too often the variables need to be measured in such tiny areas that they are not accessible to measurement, or the measurement is destructive. On the other hand, if the measurement is not made and there is an error in processing, the money spent in further work on the material is wasted.

One way to deal with this problem is to provide on each silicon wafer a small area devoted to special test structures for process-control purposes. These structures range from simple rectangular regions for measurement of electrical conduction of certain layers, or structures for checking alignment of photoresist processes, to transistor structures typical of those buried deep within an integrated circuit. Measurements routinely made on these structures can tell the process engineer much about the day-to-day reproducibility of processes and can help pinpoint the troublesome process when disaster strikes. This information more than adequately compensates for the small area of each wafer used to carry the test structures and thus not available for salable devices.

Semiconductor manufacturing processes are by no means thoroughly understood, largely because of the lack of adequate measurement methods. Most process steps involve high-purity materials, thin layers, small dimensions, low concentrations, or other extremes of conditions which make measurements difficult at best. What is needed are simple, reproducible, nondestructive measurement techniques that can be done routinely in a factory environment by technically unsophisticated people. There is a large gap between the need and what is possible at present. This unsatisfactory measurement and control situation leads to increased cost. Inaccurate measurements can make good material appear to be scrap and to be needlessly discarded, or material which is outside of established process control limits to be accepted as good. In the latter case, money will be wasted on further processing.

\section{PRODUCT DEVELOPMENT}

Successful development of a new semiconductor product requires a confluence of several factors. First, a need must be established for the product. If there is no need, there is no market, and the development is an exercise. While this seems obvious, it has not always been recognized. The need implies a set of performance specifications for the new product if it is to be successfully applied.

Given a set of characteristics, a device structure can be designed that yields at least a first approximation of these characteristics. While designs for discrete devices can occasionally be done without recourse to computer systems, the complexity of integrated circuits and the very large number of individual devices that go together to make up a single integrated circuit require the use of computer assistance. Modern integrated circuits may contain several million individual transistors in a single device; the number of devices overtaxes human abilities to position and interconnect them correctly. Since most of these devices are exact repetitions of other devices within the same integrated circuit, differing only in location and perhaps orientation, computer aid is natural. In addition, since the final set of masks to produce the product requires registration of many levels of extreme complexity, the ability of the computer to remember what exists in the other levels aids immeasurably in creating the proper form for the one being worked on at the moment. Thus registration errors between masks can be reduced. 
In addition, the particular characteristics of the processes in use by each manufacturer are different. This leads to requirements, called design rules, which apply in the preparation of the photomasks, and which are not the same for different manufacturers and even for different process sequences within the same manufacturer's house. In general, processes in use by various firms, even to produce devices with essentially identical properties, are sufficiently different that the masks used by manufacturer A will not work in manufacturer B's environment.

When the initial design is complete, and the masks are available, processing of silicon can begin. First attempts to build the product are usually carried out in a development laboratory. The products of this effort are tested and the results compared with the initial specifications for the product. Any design corrections are made, and the fabrication process is repeated. Once the design to produce the new product is established, and the process is considered well enough controlled to give satisfactory yields, manufacture of the product in the factory can begin. The transfer from laboratory to factory is fraught with difficulties, because what was previously done by highly skilled people must now be done by relatively less skilled factory workers who do not in general understand the details of what they are doing. If the new product uses the same sequence of processes as other products already being manufactured, less difficulty is usually encountered. If, however, an entirely new process sequence is required, the road to successful manufacture of a new product is studded with obstacles.

Initial yields of complex integrated circuits are frequently below 5 percent. This is obviously not satisfactory. Through continuous refinement these yields improve, leading to a final product cost that decreases sharply with time. The semiconductor industry's record of product cost reduction is probably unmatched.

Semiconductor manufacturers have always provided substantial technical assistance to their customers in the use of their products. In the beginning, when semiconductors were replacing vacuum tubes in old applications, the radically different characteristics of semiconductors created considerable difficulty in application. As a part of marketing operations, engineering assistance was extended to customers to help them to apply semiconductors correctly. With the proliferation of types of semiconductor products, this application assistance has continued to be required.

\section{PRODUCT TYPES}

In the following paragraphs each of the major kinds of semiconductor devices will be described briefly.

\subsection{Diodes}

Signal diodes are elementary one-junction small rectifiers, by definition limited to forward average currents of 100 milliamperes or less. Their reverse voltage rating is generally 200 volts or less. Typically, these devices are packaged in small glass or plastic envelopes with axial leads. They are mature products; that is, their design and manufacturing processes are stable and their sales volume is steady or decreasing with time. Many of the circuit functions formerly done by signal diodes are now performed by integrated circuits. 
An important class of signal diodes is made using a slightly different junction structure that has not been described above. If a layer of a properly selected metal is evaporated onto a silicon wafer, it is found that the metal-silicon interface possesses rectifying characteristics. This is termed a Schottky barrier. Devices utilizing this effect are called Schottky diodes; they have a very fast recovery time from the forward-conducting to the reverse-blocking mode, a feature that is important in some applications. Schottky barrier devices are made in larger sizes as well, and a small part of the rectifier market described below is supplied this way. Because of the limited reverse-blocking voltage of Schottky devices, however, the impact of this technique in the rectifier business is substantially less than it is for signal diodes.

Rectifiers are defined as simple $p$ - $n$ junction devices having two leads and carrying currents greater than 100 milliamperes. They can be very large indeed; rectifiers rated in the thousands of amperes are made and are packaged in enclosures $80 \mathrm{~mm}$ (3 inches) or more in diameter. Devices for these applications may also have very high reverse-blocking voltages, ranging up to 3,500 volts.

Still another two-terminal single-junction device is the Zener, or voltage reference, diode. These devices are designed and made to be used in the reverse-biased direction, with the voltage breakdown characteristic of the blocking junction carefully controlled. By passing a small current through the device in the reverse direction at a voltage exceeding the normal rating of an ordinary device, a very stable reference voltage drop occurs. These devices are used widely to establish fixed values of voltage in power supplies and other specialized applications.

Another specialized rectifier is the variable capacitance or varactor diode. When a $p-n$ junction is biased in the reverse direction, the free charge carriers are swept away from a region in the neighborhood of the $p-n$ junction. This produces a layer free of any mobile charge, and in every respect this layer functions as a dielectric (an insulator). A dielectric bounded on either side by a conducting medium forms a capacitor. Capacitors made in this fashion, in silicon, have the valuable feature that their capacitance can be controlled electrically. By changing the magnitude of the applied reverse voltage, the width of the blocking layer is changed and the capacitance also changes. By controlling the distribution of the dopant atoms on either side of the $p-n$ junction, a variety of voltage-capacitance characteristics can be designed. These devices have increasing applications in electronically tuned radio and television receiving equipment.

Another specialized class of $p-n$ junction diode is used for detection of light. When light is absorbed in silicon, pairs of holes and electrons are created. If this occurs in the neighborhood of a reverse biased $p-n$ junction, these holes and electrons form a current much larger than the small current that would pass through that junction in the dark. In connection with yet another specialized $p$ - $n$ junction device, the light-emitting diode, devices can be made for sensing holes in computer cards and for communicating signals between circuits at different voltages, for example.

Light-emitting diodes are not made of silicon, but of gallium-arsenic compounds. These compounds have the property that when holes and electrons recombine in a forward-biased junction, the energy that they represent appears in the form of light with a modest but useful efficiency. The wavelength of this light from pure gallium arsenide is in the near infrared and 
therefore is invisible to the human eye. If part of the arsenic is replaced by phosphorus, however, diodes made of gallium arsenide-phosphide emit light which is bright red, the familiar color of the digits on many electronic products. This same red light is readily absorbed by silicon. If, therefore, a light-emitting diode and a photodiode are arranged in proximity, current passing through the light-emitting diode in the forward direction will be detected by the photodiode as a reverse current increase. Such devices are called opto-electronic couplers. Not all optoelectronic couplers use photodiodes. Many instead use a photosensitive transistor that provides greater sensitivity to the light signals. Specialized light-emitting diodes can operate as lasers, emitting intense light in a very narrow beam. These devices are used in many applications, most familiarly for reading the information on compact disc music and video records.

Other specialized diodes are used as oscillators at ultrahigh frequencies. This class of very highfrequency signal sources includes more than one type of $p-n$ junction diode; tunnel diodes, IMPATT diodes, and Gunn effect diodes are all in this category. They all work in different ways, but the end result is similar. All generate ac signals of extremely high frequency that are useful in communications equipment in the microwave regions.

\subsection{Transistors}

All the devices heretofore discussed have two external leads. We will now consider three-leaded devices -- transistors, of which there are many specialized types. Signal transistors, in which the power dissipation is 1 watt or less, are used in great variety but in decreasing numbers, having been displaced in most applications by integrated circuits.

Larger transistors, capable of dissipating more than 1 watt of power, are classified as power transistors. They range to over 100 amperes in current-carrying capability, or up to nearly 2000 volts in voltage breakdown rating. All transistors share the common characteristic that the output current is a predictable and generally proportional function of the input current.

Included in signal and power transistor categories are two specialized devices used at frequencies above 300 megahertz. They are specialized in the sense that they are made with extremely small elements. For example, a microwave signal transistor to operate at 10 gigahertz may have lines in its metallization pattern $0.3 \mu \mathrm{m}$ wide, spaced $0.3 \mu \mathrm{m}$ apart. This very fine geometry puts extreme requirements on the photoresist processing techniques used to make these devices. Similarly, microwave power transistors also use a very large number of tiny elements in their construction to permit them to operate efficiently at very high frequencies. These devices comprise a rapidly developing segment of the semiconductor business; they are being used in high-frequency radio transmission and in cable television systems.

\subsection{Thyristors}

A device that has extremely important applications in industrial and power control equipment is the thyristor. It is sometimes referred to as the silicon-controlled rectifier, or SCR, and is used as a switching device. Its useful properties stem from the fact that when it is in its nonconducting state, only a very small current can flow, and, when it is in the conducting state, large currents can be passed with a small voltage drop. Thus, a relatively small device can switch quite 
large amounts of power. Switching is initiated by means of a current into a control terminal, called the gate. The thyristor is normally in the off-condition. When a certain amount of current is conducted into the gate, it switches into the on-condition. The gate signal needed is only a small impulse, because once switching has occurred, the gate loses control. The thyristor then remains in the on-state until the current through it is reduced to nearly zero by the external circuit. At this time the off-state is recovered, and the device remains in the off-state until another gate signal is received. Thyristors are available with current ratings as high as 2500 amperes and with voltage ratings as high as 5000 volts.

\subsection{Integrated $\underline{\text { Circuits }}$}

An integrated circuit (IC) is nothing more than a collection of individual transistors, diodes, capacitors, and resistors, all made extremely small, arrayed in a single piece of silicon and interconnected by a pattern of metallization on the surface of the die. The arrangement is made to perform a specific circuit function that may be quite simple, as in the case of a gate circuit for a computer, or that may be extremely complex and embody a large fraction of the circuitry for an entire television receiver. From the smallest to the largest integrated circuit, however, the building blocks are still the same.

The earliest types of integrated circuits were used in computers. Integrated circuits are particularly well suited to computer applications because computers are made up of large numbers of a few kinds of circuits; there may be millions of gate circuits in a computer. The earliest application of integrated circuits was simply to replace a gate circuit made of discrete parts with a gate circuit made in a single piece of silicon. Immediate economies of scale existed in this application, and integrated circuits have invaded the computer domain to the greatest degree.

Since computer circuits are generally arranged to operate in either an on-condition or an offcondition (binary circuitry), ICs used in this manner are referred to as digital circuits. Their behavior is defined only in the on- or off-state. Digital ICs are divided into families having certain characteristics in common. First, the input and output voltage swings of all devices of a family fall within the same ranges. Second, the power supply requirements are similar. Third, the relative speeds of all devices in the family are similar. These characteristics are achieved by using similar circuitry for all members of the family. Families are named according to the type of basic circuit element that is used. There are, among the obsolete families of digital circuits, such types as diode-transistor logic (DTL), resistor-transistor logic (RTL), and so on. These families have been replaced by families that are either faster, use less power, or are less expensive. Now the important bipolar digital families of ICs are transistor-transistor logic (TTL), emitter-coupled logic (ECL), and integrated-injection logic $\left(I^{2} L\right)$. Within families there are varieties that differ in speed or power consumption or both. But, for example, all TTL circuits can work together, sharing common power supply voltages and common input-output voltage levels. Interconnection between families, however, is often more difficult, although it is done when necessary. The present bipolar digital families of interest mentioned above are expected to remain the significant members of this class of device for several years to come. 
Digital circuits are further subdivided into bipolar integrated circuits, using junction transistors, or MOS (metal-oxide-silicon) circuits composed of MOS transistors. MOS digital circuits are distinguished by the fact that their power requirements are significantly lower than bipolar digital circuits, although in general their speed is also less. In many applications this is an acceptable compromise and has led to development of a variety of MOS device families. There are $p$ channel MOS ( $p$-MOS), $n$-channel MOS ( $n$-MOS), complementary MOS (CMOS), silicon-oninsulator (SOI), and charge-coupled devices (CCD).

The MOS digital circuits were developed in the order given above, $p$-MOS being oldest and CCD being newest. With the exception of CCDs, their speed of operation also has increased through the various families from MOS to SOI. The speed increase has not always been at the price of increased power consumption, however; CMOS has extremely low power consumption. As an additional feature, $C C D$ devices are also photosensitive. Linear CCDs are used to make imagesensing devices for use in TV cameras.

The low power consumption of MOS ICs made possible the pocket calculator and the electronic watch, both of which must be sparing of power to give satisfactory battery life. More subtly, low power is also important in many other applications. Since the use of ICs permits great circuit complexity to be put in a very small volume, even the modest power dissipation of ICs becomes significant when many such devices are put together into one equipment enclosure. Much the greater part of today's integrated circuit production is in MOS devices, largely for this reason.

In contrast with digital ICs in which the transistors and circuits are designed to operate in either one or the other of two states with rapid transitions between, linear integrated circuits (analog ICs) are designed to have a predictable functional relationship between input and output. For instance, in an IC amplifier, the output is a magnified version of the input. The total gain through such a circuit may be 1 million or more. Design and manufacture are more difficult for linear integrated circuits than for digital circuits, a fact which somewhat delayed their growth. Furthermore, there is a much larger variety of linear circuits than there is of digital circuits, and each variety of circuit requires a separate design.

Newer types of integrated circuits may combine both digital and linear functions, or include both bipolar and MOS circuitry, on the same chip. Similarly, an integrated circuit and a power device can be combined to provide both logic and power control either on the same chip or at least within the same package. All of these combinations make the application of the device simpler (and less expensive) for the user at some increase in complexity for the device manufacturer.

The cost of designing a complex integrated circuit may be several million dollars, so the task is not to be undertaken lightly. A substantial number of applications must exist beforehand. It should be noted that this cost covers only the design; if a new process sequence, not already in use in the factory, must be developed, the cost can easily be much greater. If the circuit is a microprocessor or microcomputer, the cost of developing the associated software will also run into the millions of dollars.

Six to eight linear integrated circuits can perform all of the functions required inside a television set with few exceptions. Linear integrated circuits are also available to make AM radios, FM 
radios, low-power stereo amplifiers, etc. These applications share the common factors of a large market and relatively standardized circuitry.

\section{APPLICATION TRENDS AND THEIR IMPACTS}

Cost and complexity trends in the industry are interesting to consider. In ICs, the average cost per component in 1961 was $\$ 1$. By 1972 , this figure was $\$ 0.01$, and in 1975 it was $\$ 0.001$. One megabit memory chips, with over 2 million transistors, now cost about $\$ 4$ in large quantities ( $\$ 0.000002$ per component). The trend for discrete devices is by no means as steep, but it is strongly downward nonetheless. This rapid decrease in cost with the passage of time has made today's personal computers economically possible.

On the other hand, complexity has grown. Where in 1959 one device per chip was the norm, by 1973 over 10,000 components per chip were possible. This increase rate corresponds closely to doubling each year. It continued at this rate for 16 years (a 65,000-fold increase by August 1975) but has slowed somewhat since that time. In 1989, development chips having about 50 million components have been described. This growth has been made possible by reduction in size per component, by increased chip size, and by ingenuity of device and circuit design.

The net effect of these trends has been to reduce substantially the cost of performing electronic functions. This change appears in products in a variety of ways:

1. Transistor radios, pocket calculators, and electronic watches have each, on their own time scale, been available at rapidly falling prices limited ultimately by the material and labor costs of the whole product. The electronics part of this total cost is relatively modest.

2. Television sets, major appliances, personal computers, and automobiles tend to incorporate more and more sophistication at a relatively constant price. Communication and industrial control equipment also shares this trend. The electronics content of these items tends to increase with time to provide the added sophistication.

3. The size of the largest practical electronics systems keeps increasing. Not only do decreasing costs per component permit more components to be used in an affordable system, but the failure rate per component in an IC has dropped at a rate almost identical to the rate of increase in the number of components per IC. The failure rate per IC package has not significantly changed. Practical system size tends to be limited more by the tolerable failure rate of the system.

In power semiconductors, the trends are different in kind but similarly broadening in their impact on our daily life. Applications of power devices are most apparent in variable lighting controls, speed controls on power tools and appliances, and in other major household appliance control functions. Less visible to the consumer but of more economic importance are industrial controls that are at the heart of many machine tools and that operate many industrial processes. The economic effect stems from the improved productivity that is provided by these sophisticated kinds of plants and machinery. 
The growing concern for our energy sources has caused the development of many electric powergenerating schemes, such as solar cells and windmills, whose output is either direct current or perhaps alternating current of varying frequency. The output from these sources requires modification to be compatible with the existing constant-frequency ac power distribution system, and solid-state power-converting equipment will be necessary.

A final example is the use of power semiconductor devices as the "muscle-power" to operate mechanical systems under the primary control of electronic logic circuits. Such systems are already in use in antiskid controls on vehicles and for a variety of other vehicular subsystems. The need for power solid-state devices to do the work, under control of logic circuits (computers) that make decisions, is not unique to the automotive field, of course, but the example illustrates the concept.

\section{SUMMARY}

We have explored briefly the wide range of technologies used in the semiconductor industry to transform simple, pure crystals of a few kinds into a constantly growing array of solid-state devices and circuits. These have also been described. The manufacturing process is materialsand process-oriented, requiring a wide variety of technical professionals for its conception, development, and implementation. It is an industry of extremes: highest purity, smallest linewidths, most complex products, simple concepts, and bewildering processes. In the 42 years since the transistor was invented, a $\$ 50$ billion annual flood of semiconductors worldwide has radically changed the electronics industry and our daily lives. Semiconductor devices are at the heart of every electronic product and are the indispensable components in the $\$ 248$ billion worth of electronic goods manufactured in 1988 in the United States alone.

Semiconductor manufacturing operations are limited at any particular time by materials, processing, and measurement problems. As today's problems are solved and new progress is made, new problems arise and impose new limits. The past performance of the industry in controlling processes, improving yields, and raising reliability levels has resulted in dramatic reductions in cost. In turn, lowered cost has allowed penetration of electronic products into new areas. Fundamental physical limits will ultimately determine how complex an integrated circuit can become, for example, but there is still a long way to go. 


\section{APPENDIX A. Units of Measurement}

The semiconductor industry, being international in scope, uses metric measures to a great extent. Most Americans are not yet accustomed to thinking in these units, and they especially do not have an intuitive feeling for very small dimensions expressed in metric terms. This discussion is meant to relate metric linear measures to familiar objects.

A nickel is about 2 centimeters in diameter. 1 centimeter $(\mathrm{cm})=0.3937$ inch.

The diameter of paper-clip wire is about 1 millimeter. 1 millimeter $(\mathrm{mm})=0.1 \mathrm{~cm}$.

A human hair is about 100 micrometers in diameter, and cigarette paper is about 25 micrometers thick. 1 micrometer $(\mu \mathrm{m})=0.001 \mathrm{~mm}$ (one-thousandth $\mathrm{mm}$ ).

The wavelength of green light is about $0.5 \mu \mathrm{m}$. This dimension is close to that of the finest detail that can be seen using a high-powered light microscope.

A nanometer $(\mathrm{nm})$ is $0.001 \mu \mathrm{m}$. Atoms in solids are typically about $0.5 \mathrm{~nm}$ apart. The thinnest oxide films used in integrated circuits are about $10 \mathrm{~nm}$ thick, or about 20 atoms.

There are no familiar objects in the size range from 1 to $25 \mu \mathrm{m}$ simply for the reason that things so small cannot be seen by the unaided eye; yet, this is just the region of dimensions of many of the parts of integrated circuits. 


\section{APPENDIX B. $\underline{\text { How }} \underline{\text { silicon }} \underline{\text { is }}$ obtained $^{3}$}

Silicon dioxide in its crystalline form is quartz, a very common mineral. Many sand deposits and beaches are almost entirely composed of quartz grains, the raw material for making silicon, though only a few of these deposits are sufficiently pure for doing so. The sand is mixed with carbon and decomposed in an electric furnace to produce carbon monoxide and a metallurgical grade of silicon which is about 98 percent pure. This material is used in metallurgy for deoxidizing steels and strengthening aluminum alloys. Only about 1 percent of the world's production of metallurgical grade silicon is further refined to produce semiconductor-grade silicon.

Metallurgical silicon is reacted chemically with hydrogen chloride to produce trichlorosilane $\left(\mathrm{SiHCl}_{3}\right)$ and other gaseous silicon-hydrogen-chlorine compounds. The trichlorosilane is separated from the other compounds and purified by distillation. With great care, it is made extremely pure.

The trichlorosilane is decomposed by heat in the presence of hydrogen on slender silicon rods heated by the passage of electric current. Silicon deposits on the rods and hydrogen chloride is produced, to be recycled back to the beginning of the process to treat more metallurgical silicon. The deposited silicon is polycrystalline, with an impurity content of only a few parts per trillion, and is used in the crystal-growing processes mentioned earlier. The entire process is quite energy-intensive, consuming over 300 kilowatt-hours of electricity per kilogram ( 2.2 pounds) of polysilicon produced.

${ }^{3}$ A more detailed description may be found in T. Abe, "Crystal Fabrication," in VLSI Electronics: Microstructure Science, vol. 12, pp. 3-61, Academic Press, New York (1985). 
Aligner, mask

Aligners

types of 11

contact 11

projection 11

reduction 11

Annealing

step-and-repeat 11

to remove implantation damage 14

Assembly

into completed parts 16

of low-power devices 16

of power devices 16

Bias, forward

definition 4

Bias, reverse

definition 4

Boron

doping effects 2

Complexity of integrated circuits

trends in 24

Compound semiconductors

significance 2

Connections

electrical 3,14

Cost trends

in integrated circuits 24

Crystal

single 7

Crystal, single

processes to produce 7

Czochralski

growth method for single crystals 7

Defects size of crystals produced by 7

cause of yield loss 11

in photopatterns 11

Deposition, chemical vapor

making oxide films by 10

Development

of semiconductor products 18

Device design

need for computer aid 18

Diameter 
production of controlled 9 standardized 9

Dicing

into individual chips 15

Diffusion

$$
\begin{aligned}
& \text { doping by } 13 \\
& \text { problems related to } 10
\end{aligned}
$$

Diodes

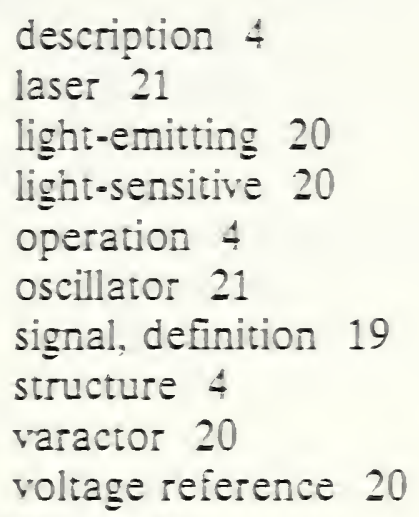

Dopant elements

addition of 3

electrical effects 2

Doping

of selected areas 10

Electrical connection 14

Electron-beam

direct writing on wafer by 12

Electron-beam pattern generation

process of 11

Epitaxy

definition 9

process 9

properties of layers grown by 9

purposes of 9

Equipment

characteristics 6

final testing 17

makers 6

relation to process technology 6

Feature size

limitations on 12

Float-zone

absence of oxygen in 8

crystal growth method 8

sizes of crystals available 8

Germanium 2

limitations 2 
Insulating layers

uses 3

Integrated circuits

complexity trends in 24

cost trends 24

description 22

effects on system cost 24

use in computers 22

Integrated circuits, digital

bipolar 23

families of 22

MOS 23

Integrated circuits, linear

description 23

Ion implantation

damage caused by 14

description of 13

Measurement

difficulties of 18

impact on cost 18

key to process control 18

using test structures 18

Metal deposition

Metal films

forming patterns of 10

formation of 15

Metallization 14

Metric units of measure

description in familiar terms 26

Micropatterning

Mounting

processes for 10

of chips into packages 16

N-type

origin of electrons 2

Neutron irradiation

method of doping silicon 8

Oxidation, thermal

process of 10

Oxygen

absence in float-zone silicon 8

in Czochralski-grown silicon 7

p-n junction

definition 3

P-type

origin of holes 3 


\section{Package}

Passivation

$$
\text { protective } 17
$$

for chip protection 14

materials used for 14

Pattern generator

use of 11

Patterns

alignment of 11

defects in 11

formation of 10

Patterns, master

creation by electron-beam process 11

creation of 10

optical generation of 11

use of 10

Phosphorus

doping effects 2

Photomasking

alignment of patterns in 11

Photomasking process

problems in 12

Photomasks

defect reduction in 12

defects in 12

for $x$-ray lithography 13

relative sizes of 11

repair of 12

typical number required 11

Photoresist

for $x$-ray lithography 13

use of 10

Polycrystalline

concept 7

effects of 7

Power semiconductors

applications 24

Printers

contact 11

Process control 17

use of test structures in 18

Professional skills

types used 1

Purity

of materials 4

Rectifiers 
definition 20

Schottky diode

properties 20

Scribing

into separate chips 15

methods of 15

Semiconductor materials

Silicon

properties 2

crystal structure 2

electrical conductivity 2

manufacturing process 27

purity 3

reasons for use 2

source of raw material 27

Silicon dioxide

deposition of 10

formation of 10

importance 2

selective removal of 10

thickness of 10

Silicone

distinction from silicon 7

Steppers, wafer 12

Synchrotron

as $\mathrm{x}$-ray source 12

cost of 13

Technical assistance

to customers 19

Temperature, high

effects of in dense integrated circuits 10

Test structures

description 18

Testing 15

equipment 15

final, equipment for 17

for cost control 15

Testing, final 17

Thyristors

properties 21

Transistor

operation 5

structure 5

Transistors

high-frequency 21

Transistors, power 
definition 21

Transistors, signal

definition 21

Value

of monocrystalline silicon 9

Wafers

cleaning 9

cutting of, from crystal 9

edge finishing 9

etching and lapping 9

polishing 9

Wire bonding

process of 16

$\mathrm{X}$-ray lithography

need for 12

$\mathrm{X}$-ray sources

brightness of 12

types of 12

$\mathrm{X}$-rays

Yield

use of synchrotron for producing 12

in mature process 11

level in early manufacturing 11

Yield loss

from pattern defects 11 


.




\begin{tabular}{|c|c|c|}
\hline & $\begin{array}{lr}\text { UST-114A } & \text { U.S. DEPARTMENT OF COMMERCE } \\
\text { REV. 3-90) } & \text { NATIONAL INSTITUTE OF STANDARDS AND TECHNOLOGY }\end{array}$ & $\begin{array}{l}\text { 1. PUBLCATION OR REPORT NUMBER } \\
\text { NIS T I R } 4414\end{array}$ \\
\hline & & 2. PERFORMINO ORGANIZATION REPORT NUMBER \\
\hline & BIBLIOGRAPHIC DATA SHEET & \\
\hline & & 3. PUBUCATION DATE \\
\hline & & SEPTEMBER 1990 \\
\hline & TITLE AND SUBTITLE & \\
\hline & $\begin{array}{l}\text { Semiconductor Technology for the Non-Technologist } \\
\text { second edition }\end{array}$ & \\
\hline & AUTHOR(S) & \\
\hline & R. I. Scace & \\
\hline 6. & PERFORMING ORGANIZATION (IF JOINT OR OTHER THAN NIST, SEE INSTRUCTIONS) & 7. CONTRACT/GRANT NUMBER \\
\hline & $\begin{array}{l}\text { U.S. DEPARTMENT OF COMMERCE } \\
\text { NATIONAL INSTITUTE OF STANDARDS AND TECHMOLOQY }\end{array}$ & none \\
\hline & QAITHERSBURG, MD 20899 & $\begin{array}{l}\text { 8. TYPE OF REPORT AND PERIOD COVERED } \\
\mathrm{N} / \mathrm{A}\end{array}$ \\
\hline
\end{tabular}

9. SPONSORING ORGANIZATION NAME AND COMPLETE ADDRESS (STREET, CITY, STATE, ZIP)

10. SUPPLEMENTARY NOTES

11. ABSTRACT (A 200-WORD OR LESS FACTUAL SUMMARY OF MOST SIGNIFICANT INFORMATIOH. IF DOCUMENT INCLUDES A SIGNIFICANT BIBUOGRAPHY OR UTERATURE SURVEY, MENTION IT HERE.)

The properties of semiconductor materials, the methods of processing them, and the solidstate products made from them are described in terms intended to be understandable by the lay person. The semiconductor industry has grown at a rate of 21 percent per year compounded for the last twenty years, and its products have declined in unit cost by a factor of five in current dollars (a factor of ten in constant dollars) in the same period. This very satisfactory but anomalous behavior has attracted the interest of many who are not familiar with the technology of the industry, yet who need to have some understanding of it. This report is intended to help meet that need.

12. KEY WORDS (6 TO 12 ENTRIES; ALPHABETICAL ORDER; CAPITALLZ ONLY PROPER NAMES; AND SEPARATE KEY WORDS BY SEMICOLONS)

Integrated circuits; semiconductors; semiconductor devices; semiconductor processes; semiconductor technology; silicon.

\section{AVAILABIUTY}

\section{$\mathrm{X}$ UNUMITED}

FOR OFFICIAL DISTRIBUTION. DO NOT RELEASE TO MATIOMAL TECHMICAL INFORMATION SERVICE (NTIS).

ORDER FROM SUPERINTENDENT OF DOCUMENTS, U.S. COVERMMENT PRINTING OFFICE, WASHINGTOH, DC 20402

ORDER FROM MATIOMAL TECHMICAL IMFORMATION SERVCE (NTIS), SPRIMOFIED, VA 22161.
14. NUMBER OF PRINTED PAGES

$$
38
$$

15. PRICE

$\mathrm{A} 03$ 


